\title{
Evaluation of the Antimicrobial Activity of Silver Nanoparticles on Antibiotic-Resistant Pseudomonas aeruginosa
}

\author{
Aliakbar Nasiri', Roghayeh Afsar Gharebagh ${ }^{2}$, Seyed Ali Nojoumi ${ }^{3}$, Majidreza Akbarizadeh ${ }^{4}$, Sharareh \\ Harirchi $^{5}$, Masoud Arefnezhad ${ }^{6}$, Shahla Sahraei ${ }^{7}$, Mehran Hesaraki ${ }^{4}$, Mahdi Afshari ${ }^{6}$, Fereshteh Javadian ${ }^{6 *}$, \\ Maryam Sheykhzade Asadi ${ }^{8}$, Zahra Shahi ${ }^{8}$, Aliyeh Sargazi ${ }^{9}$
}

\begin{abstract}
${ }^{1}$ Assistant Professor of Anesthesiology, Department of Anesthesiology, Zabol University of Medical Sciences, Zabol, Iran ${ }^{2}$ Assistant Professor of Cardiology, Department of Cardiology, Urmia University of Medical Sciences, Urmia, Iran ${ }^{3}$ Assistant Professor of Microbiology, Microbiology Research Center, Pasteur Institute of Iran, Tehran, Iran ${ }^{4}$ Assistant Professor of Pediatrics, Department of Pediatrics, Zabol University of Medical Sciences, Zabol, Iran ${ }^{5} \mathrm{PhD}$ Student of Microbiology, Department of Microbiology, Isfahan University of Medical Sciences, Isfahan, Iran ${ }^{6}$ Zabol University of Medical Sciences, Zabol, Iran

${ }^{7}$ Zabol Medicinal Plants Research Center, Zabol University of Medical Sciences, Zabol, Iran

${ }^{8}$ Kerman Science and Research Branch, Islamic Azad University, Kerman, Iran

${ }^{9}$ Student Research Committee, Zabol University of Medical Sciences, Zabol, Iran
\end{abstract}

\section{*Correspondence to Fereshteh Javadian Email: \\ fereshteh.javadian@yahoo.com}

Published online 29 June 2016

Please cite this article as follows: Nasiri A, Afsar Gharebagh R, Nojoumi SA, et al. Evaluation of the antimicrobial activity of silver nanoparticles on antibiotic-resistant Pseudomonas aeruginosa. Int J Basic Sci Med. 2016;1(1):2528. doi:10.15171/ ijbms.2016.06.

\begin{abstract}
Background: Antimicrobial resistance is one of the major characteristics of infectious agents. Silver nanoparticles (AgNPs) have been introduced as novel antibacterial agents in accordance with the traditional treatments. Our purpose of this study was to evaluate the antimicrobial activity of AgNPs on the Pseudomonas aeruginosa (P. aeruginosa) that are resistant to antibiotics. Methods: During a cross-sectional study, we tried to evaluate 20 strains of $P$. aeruginosa isolated from the urine cultures of patients admitted to the hospital due to urinary tract infections. The AgNPs were commercially purchased. The minimum inhibitory concentration (MIC) of AgNPs in different concentrations was determined by the dilution in wells on bacteria. The antibiotic susceptibility pattern of $P$. aeruginosa was evaluated by the Kirby-Bauer disk diffusion standard. Results: Current study indicated that $P$. aeruginosa were resistant to four types of agents including ampicillin (85\%), nitrofurantoin (65\%), nalidixic acid (65\%), and ciprofloxacin (15\%) and result of nanosilver indicated that the most MIC was $100 \mathrm{ppm}$ concentration, and six strains of $P$. aeruginosa were inhibited by it.

Conclusion: Our study presented a new type of silver nanoparticle and indicated that they can be embedded in bone cement to prevent infections once synthetic conditions are tailored for such applications.
\end{abstract}

Keywords: Nanosilver, Antimicrobial activity, Pseudomonas aeruginosa, Minimum inhibitory concentrations (MIC), Antibiotic-resistant

\section{Introduction}

In recent decades, several studies have been carried out regarding the transport of materials, particularly precious metal particles in the nano regime. Silver nanoparticles (AgNPs) show special properties that are great scientific achievements in nanotechnology. Their use is widespread as they are produced in different ways depending on the type of material and its applications. Nanoparticles are among the most common elements in science and nanotech-
\end{abstract}

nology. They hold interesting properties; in this regard, they have a wide variety of applications in the chemical, medical, and pharmaceutical industries, as well as electronics and agriculture. In their chemical composition, these particles are composed of metal, ceramic, polymer, and semiconductor. Metals such as silver are considered safe and are effective antibacterial agents that can kill more than 650 types of microorganisms including bacteria and virus. By increasing the surface to volume

Copyright (C) 2016 The Author(s); Published by Zabol University of Medical Sciences. This is an open-access article distributed under the terms of the Creative Commons Attribution License (http://creativecommons.org/licenses/by/4.0), which permits unrestricted use, distribution, and reproduction in any medium, provided the original work is properly cited. 
ratio, the nanoscale silver antibacterial properties can be improved. ${ }^{1}$ AgNPs are produced usually based on optical methods, ${ }^{2,3}$ restoring either the chemical ${ }^{4}$ or silver oxide. ${ }^{5}$ Like other technologies, nanotechnology can be followed by several disadvantages; for example, experts do not have enough information in this case, and whether the destructive effects of AgNPs pertain to cells and tissues. It is possible that nanoparticles may have serious consequences for the environment and human health. ${ }^{6}$ P. aeruginosa is a gram-negative bacterium that is usually seen alone or in short chains. Colonies of these bacteria are smooth and rounded, often in green fluorescent dye that is used to identify these bacteria. In the regimens, a drug is not used alone and penicillin for example is usually used in combination with an amino glycoside like gentamicin.? The aim of this study was to investigate the antimicrobial effect of AgNPs against $P$. aeruginosa that are resistant to antibiotics.

\section{Methods}

Bacterial Strains

All 20 strains of $P$. aeruginosa were isolated from the urine cultures of hospitalized patients with urinary tract infections. The cultures were grown on nutrient agar. To identify different kinds of pseudomonas from tests, gram staining, catalase, oxidize, glucose tests, OF (oxidation fermentation), and TS (triple sugar iron) were used. ${ }^{8} P$. aeruginosa were detected from urine specimens, transferred to microbiology laboratory, using Gram-Negative Identification Panel Type II (Dade International Inc., West Sacramento, Calif.). Microdilution broth testing (MicroScan; Dade International Inc.) was applied to test the susceptibility.

\section{Agar disk diffusion assay}

Susceptibility of all antibiotics was assessed using the disc diffusion method on the Muller-Hinton agar recommended by the Clinical and Laboratory Standards Institute (CLSI). ${ }^{9}$ Staphylococcus aureus (S. aureus) isolated plates were grown overnight on the blood agar and the colony suspension was prepared using sterile saline water equivalent to a $0.5 \mathrm{McF}$ arland standard. The suspension $(100 \mu \mathrm{L})$ was spread over media plate and antibiotic disc was transferred on the aseptic surface of this inoculated plate. The isolated plates were then tested with different antibiotics viz ampicillin, nitrofurantoin, nalidixic acid, and ciprofloxacin, and their concentrations were illustrated in parenthesis.

Minimum Inhibitory Concentration and Minimum Bactericidal Concentration of Nanosilver

AgNPs powder used in this study was manufactured by Thermolon Korea Inc., according to broth microdilution method. Then the serial doubling dilutions of AgNPs were prepared in a 96-well $\mu \mathrm{L}$ plate ranged from $12.5 \mathrm{ppm}$ to $200 \mathrm{ppm}$. Ten microlitter of the indicator solution and $10 \mu \mathrm{L}$ of the Mueller Hinton Broth were added to each well. Finally, $10 \mu \mathrm{L}$ of the bacterial suspension $\left(10^{6} \mathrm{CFU} /\right.$
$\mathrm{mL})$ was added to each well to obtain a concentration of $10^{4} \mathrm{CFU} / \mathrm{mL}$. Plates were wrapped loosely with clingfilm to ensure that bacteria did not get dehydrated. Moreover, plates were prepared in triplicates and were placed in an incubator at $37^{\circ} \mathrm{C}$ for $18-24$ hours. Color change was then assessed visually. The lowest concentration at which color change occurred was taken as minimum inhibitory concentration (MIC) value. MIC is defined as the lowest concentration of extract at which microorganism does not demonstrate visible growth. Microorganism growth was indicated by turbidity. Minimum bactericidal concentration (MBC) was defined as the lowest concentration of nanosilver at which the incubated microorganism was completely killed. Microorganism growth was indicated by turbidity and P. aeruginosa ATCC 27853 was the positive control in this study.

\section{Statistical Analysis}

Results were described as mean or frequency. Comparing the mean values among groups was performed using oneway analysis of variance. All data analyses were conducted using SPSS version 19.0 software. $P$ value less than 0.05 was considered statistically significant.

\section{Results}

The average size of AgNPs was $20 \mathrm{~nm}$. The results showed that isolated pseudomonas were respectively resistant to antibiotics; however, overall $P$. aeruginosa were resistant to four types of the agents including ampicillin (85\%), nitrofurantoin (65\%), nalidixic acid (65\%), and ciprofloxacin $(15 \%)$ (Table 1). The result of nanosilver showed that the most MIC was 100 ppm concentration, and six strains of pseudomonas were inhibited (Table 2), and the least MIC was 12.5 ppm concentration, and one strain of Pseudomonas was inhibited. We also found that the antifungal effects were increased by increasing the concentration of the nanosilver. No significant difference was observed between the Inhibition of bacterial growth, regarding the nanosilver concentration $(P<0.05)$.

\section{Discussion}

Since a long time ago, scientists have detected the antimi-

Table 1. Percentage of Antimicrobial Susceptibility of 20 Strains of P. aeruginosa

\begin{tabular}{lllll}
\hline & AM & Fm & NA & CP \\
\hline S & 0 & 60 & 10 & 60 \\
$\mathrm{I}$ & 15 & 20 & 25 & 25 \\
$\mathrm{R}$ & 85 & 20 & 65 & 15 \\
\hline
\end{tabular}

Abbreviations: $\mathrm{CP}$, ciprofloxacin; $\mathrm{NA}$, nalidixic acid; $\mathrm{Fm}$, nitrofurantoin; AM, ampicillin; S, sensitive; I, intermediate; R, resistant.

Table 2. MIC and MBC of Nanosilver Against $P$. aeruginosa

\begin{tabular}{lllllll}
\hline & $\mathbf{3 . 1}$ & $\mathbf{6 . 2 5}$ & $\mathbf{1 2 . 5}$ & $\mathbf{2 5}$ & $\mathbf{5 0}$ & $\mathbf{1 0 0}$ \\
\hline MIC & 0 & 0 & 5 & 10 & 55 & 30 \\
MBC & 0 & 0 & 0 & 5 & 10 & 70 \\
\hline
\end{tabular}

Abbreviations: MIC, minimum inhibitory concentration; MBC, Minimum bactericidal concentration. 
crobial properties of silver. ${ }^{10}$ However recently, just due to the form of nanoparticles, their surface area has increased by more than $99 \%$ and therefore their antimicrobial properties have increased. ${ }^{11}$

In this study, the size of AgNPs was $20 \mathrm{~nm}$ and in 100 ppm nanosilver, the percentage of bacterial reduction was $30 \%$, while in $50 \mathrm{ppm}$ that more nanosilver was used, the percentage decreased to $55 \%$.

Haji Mirzababa et $\mathrm{al}^{12}$ showed that the nylon carpet was stained with $25 \mathrm{ppm}$ nanosilver and the percentage of bacterial reduction was $72.3 \%$, but in cases where the floor was covered with $50 \mathrm{ppm}$ that more nanosilver was used, the percentage decreased to $99.99 \%$ of bacteria. In addition, checking the stability of AgNPs on samples was standard and 1 to 10 times more stable after washing.

Chahar and Khodaday ${ }^{13}$ showed that AgNPs synthesis in the fruit juice shows maximum absorption at $420 \mathrm{~nm}$. Moreover, the antimicrobial activities of AgNPs against E. coli, S. aureus, P. aeruginosa, and B. sabtilis have been proven.

Alizadeh et $\mathrm{al}^{14}$ showed that even low concentrations of AgNPs can inhibit the Brucella melitensis. The MIC and minimum lethal concentration of AgNPs in broth macrodilution were reported as $6 \mathrm{ppm}$ and $4 \mathrm{ppm}$, respectively. In addition, anti-brucella effects of AgNPs have been observed in a mouse model.

Hernández-Sierra et $\mathrm{al}^{15}$ demonstrated that AgNPs in comparison to zinc oxide and gold nanoparticles prevent the growth of streptococcus mutants with a lower concentration.

Sintubin et $\mathrm{al}^{16}$ showed that the lowest concentration of an antimicrobial silver nanoparticle for Staphylococcus aureus was $20 \mathrm{mg} / \mathrm{mL}$.

In the present work, $P$. aeruginosa were resistant to four types of agents including ampicillin (85\%), nitrofurantoin (65\%), nalidixic acid (65\%), and ciprofloxacin (15\%).

Although large national surveys provide critical information about resistance situations, they do not address the potentiality of much higher rates of resistance within individual communities and hospitals. For example, during 2001 and 2006, rates of nonsusceptibility among the $P$. aeruginosa isolates in Brooklyn, NY ranged from $27 \%$ to $29 \%$ for cefepime, $30 \%$ to $31 \%$ for imipenem, $23 \%$ for meropenem, and $41 \%$ to $44 \%$ for ciprofloxacin. ${ }^{17}$

According to the results of the study carried out by Nikokar et al, ${ }^{18}$ it was indicated that from 182 patients admitted in burn center, 86 (47\%) had P. aeruginosa in their isolated samples with the following resistance situations: cloxacillin (91.8\%), cotrimoxazole (86\%), cephazolin $(83.7 \%)$, carbenicillin $(74.4 \%)$, piperacillin $(69.9 \%)$, ceftazidime $(68.8 \%)$, ciprofloxacin $(66.3 \%)$, tobramycin $(58.2 \%)$, amikacin $(48.8 \%)$, and gentamicin $(37.2 \%)$. The least resistance was detected for imipenem as $23.3 \%$. Out of them, $39(45.3 \%)$ isolates were detected as multi-drug resistant. Investigation by PCR reaction showed that 37 (43\%) of the P. aeruginosa isolates and 27 (69.2\%) of the multi-drug resistant strains harbored class 1 integrons. A significant relationship was observed between the pres- ence of integrons and the resistance against imipenem, ceftazidime, piperacillin, and ciprofloxacin $(P<0.001)$. Ahmadi et al $^{19}$ reported that $P$. aeruginosa strains had the highest levels of resistance against ampicillin (93\%), gentamycin $(89.5 \%)$, ciprofloxacin $(82.5 \%)$, and amikacin $(77.3 \%)$. The most effective drugs were meropenem (2.3\%), imipenem $(2.9 \%)$, polymyxin B $(21.5 \%)$, and cotrimoxazole (31.9\%).

Moazami-Goudarzi and Eftekhar ${ }^{20}$ showed $99.2 \%$ resistance to carbenicillin, $98.4 \%$ to ticarcillin, $96.2 \%$ to ciprofloxacin, $95.4 \%$ to co-trimoxazole, $94.7 \%$ to imipenem and meropenem, $93.9 \%$ to piperacillin, $93.2 \%$ to azetronam, $92.4 \%$ to tobramycin, and $87.2 \%$ to piperacillin-tazobactam. Totally, $100 \%$ of the isolates showed multidrug-resistance (resistant to more than 3 classes of antibiotics such as imipenem).

Fazeli and Momtaz ${ }^{21}$ showed that the most fluoroquinolone resistant bacteria were encoding the gene gyrA (15. $68 \%)$. They also observed high level of resistance to penicillin (100\%), tetracycline $(90.19 \%)$, streptomycin (64.70\%), and erythromycin $(43.13 \%){ }^{21}$

\section{Conclusion}

Our study provided evidence regarding the antimicrobial characteristics of a new type of silver nanoparticle which can be effective in protection against infections due to different organs.

\section{Ethical Approval \\ Not applicable.}

\section{Competing Interests}

Authors declare that they have no competing interests.

\section{Acknowledgements}

The authors would like to thank Research Center of Agricultural Biotechnology.

\section{Reference}

1. Nien Chung Y, Ming Chu P, inventors. Nanosilververcontaining preservation articles, and the preparation process and the uses thereof. Ellicott City MD US, International patent AA01N2512FI. 2007

2. Liu SX, Qu ZP. A mechanism for enhanced photocatalytic activity of silver-loaded titanium dioxide. Catalysis Today. 2004;93-95:877-884. doi:10.1016/j.cattod.2004.06.097.

3. Prucek A, Kvitek L. Silver colloid nanoparticles: Synteis, characterization, and their antibacterial activity. J Phys Chem. 2006;110(33):16248-16253. doi:10.1021/jp063826h.

4. Sondi I, Goia DV. Preparation of highly concentrated stable dispersions of monodispersed silver nanoparticles. J Colloid Interface Sci. 2003;260(1):75-81. doi:10.1016/ s0021-9797(02)00205-9.

5. Brody A, Bugusu B. Innovative food packaging solutions. J Food Sci. 2008;73(1):107-116.

6. Hogstrand C, Wood CM. The toxicity of silver to marine fish. USA: University of Kentucky; 1996.

7. Driscoll JA, Brody SL, Kollef MH. The epidemiology, pathogenesis and treatment of Pseudomonas aeruginosa infections. Drugs. 2007;67:351-368. doi:10.2165/00003495- 
200767030-00003

8. Finegold SM, Baron EJ, Bailey WR. Bailey and Scott's Diagnostic Microbiology. 8th ed. St. Louis, MO: Mosby: 1990:398-399.

9. M100-S16 Performance standards for antimicrobial susceptibility testing; sixteenth informational supplement. Wayne PA, USA: Clinical and Laboratory Standards Institute; 2006.

10. Li WR, Xie XB, Shi QS, Duan SS, Ouyang YS, Chen YB. Antibacterial effect of silver nanoparticles on Staphylococcus areus. Biometals. 2011;24(1):135-141. doi:10.1007/s10534-010-9381-6.

11. Sotiriou GA, Pratsinis SE. Antibacterial activity of nanosilver ions and particles. J Environ Sci Technol. 2010;44(14):5649-54.

12. Haji Mirzababa H, Montazer M, Rahimi MK. Evaluation of antimicrobial effects of nano-silver coated nylon carpets. Medical Science Journal of Islamic Azad University 2011; 21(2):101-107.

13. Chahar D, Khodaday AM. Silver nanoparticles produced by organic synthesis using Oak fruit and the antimicrobial activity against the causes of nosocomial infections. Journal of Ilam University of Medical Sciences 2014:21(1): 33-27.

14. Alizadeh H, Salouti M, Shapoori R, Abdollahzadeh C, Naderian C. Antibacterial effects of silver nanoparticles against Brucella Brvslamly M16 in vitro, in animal models. Journal of Medical Sciences. 2011;6(3):70-64.

15. Hernández-Sierra JF1, Ruiz F, Pena DC. The antimicrobial sensitivity of Streptococcus mutans to nanoparticles of silver, zinc oxide and gold. Nanomedicine. 2008;4(3):237240. doi:10.1016/j.nano.2008.04.005.

16. Sintubin L, De Gusseme B, Van der Meeren P, Pycke BF, Verstraete $\mathrm{W}$, Boon $\mathrm{N}$. The antibacterial activity of biogenic silver and its mode of action. Appl Microbiol Biotechnol. 2011;91(1):153-162. doi: 10.1007/s00253-011-3225-3.

17. Landman D, Bratu S, Kochar S, et al. Evolution of antimicrobial resistance among Pseudomonas aeruginosa, Acinetobacter baumannii and Klebsiella pneumoniae in Brooklyn, NY. Chemother.2007;60:78-82. [PubMed]

18. Nikokar I, Tishayar A, Flakiyan Z, et al. Antibiotic resistance and frequency of class 1 integrons among Pseudomonas aeruginosa, isolated from burn patients in Guilan, Iran. Iran J Microbiol. 2013;5(1):36-41.

19. Ahmadi K, Hashemian AM, Pouryaghobi SM, Akhavan R, Rozmina Sand Bolvardi E. Antibiotic resistance properties of Pseudomonas aeruginosa isolated from cases of superficial infections at the emergency unit. Jundishapur J Microbiol. 2016;9(1):e27646. doi:10.5812/jjm.27646.

20. Moazami-Goudarzi S, Eftekhar F. Assessment of carbapenem susceptibility and multidrug-resistance in Pseudomonas aeruginosa burn isolates in tehran. Jundishapur J Microbiol. 2013;6(2):162-165. doi:10.5812/ jjm.5036

21. Fazeli N, Momtaz H. Virulence gene profiles of multidrugresistant Pseudomonas aeruginosa isolated from iranian hospital infections. Iran Red Crescent Med J. 2014;16(10): e15722. doi:10.5812/ircmj.15722. 